\title{
Monkeys and mountains in Yunnan, China
}

\author{
Tetsuro Matsuzawa ${ }^{1,2,3}$
}

Published online: 30 May 2017

(C) Japan Monkey Centre and Springer Japan 2017

This article aims to report the recent research activity carried out by my colleagues and me, in Yunnan, China. The main focus was to better understand the snub-nosed monkeys and the mountainous region that is their home. Snub-nosed monkeys live at high altitudes, about 3000-4000 m, and sometimes even higher; a region covered by snow throughout the winter season. As a keen mountaineer (Matsuzawa 2016), I have followed the research on snub-nosed monkeys living in high snowy mountains with close attention. Golden snub-nosed monkeys (Rhinopithecus roxellana), have been investigated by Li Bao-Guo and his colleagues for decades (Qi et al. 2014; Zhang et al. 2006). In Yunnan, another species, called the Yunnan snub-nosed monkey (Rhinopithecus bieti), has been studied by Long Yong-Cheng and his colleagues, also for a considerable length of time (Long et al. 1994). Yunnan attracts me because it has unique monkeys in high snowy mountains, located at the east end of the Great Himalaya Range.

Yunnan is a province of China, but the area it covers, 394,100 square $\mathrm{km}$, is almost equal to that of Japan. The capital is Kunming, nicknamed the Spring city. While Japan is surrounded by sea, Yunnan is landlocked, surrounded by Tibet, Sichuan, Guizhou, Guangxi Zhuang

Electronic supplementary material The online version of this article (doi:10.1007/s10329-017-0613-z) contains supplementary material, which is available to authorized users.

Tetsuro Matsuzawa

matsuzawa.tetsuro.8w@kyoto-u.ac.jp

Institute for Advanced Study, Kyoto University, Kyoto, Japan

2 Primate Research Institute, Kyoto University, Inuyama, Japan

3 Japan Monkey Centre, Inuyama, Japan
Autonomous Region, Vietnam, Laos, and Myanmar. The highest point of Yunnan is Meili Xue Shan (Meili Snow Mountain, $6740 \mathrm{~m}$ ) located in the northwest of the province. The latitude of Yunnan is about equal to that of Taiwan. The southern part, called Xishuangbanna, lies in a subtropical area in which you can see elephants and other tropical creatures.

My commitment to Yunnan goes back to 1990. I reached the summit of Shishapangma (Xixabangma, $8027 \mathrm{~m}$ ), Tibet, on May 17, 1990. Shishapangma is one of only 14 peaks above $8000 \mathrm{~m}$ in the world. The Academic Alpine Club of Kyoto (AACK) sent a joint Japan-China expedition to Shishapangma and succeeded in supporting 22 climbers to the summit, including the first Chinese Han woman (http://www.aack.info/ja/archive/Xixabangma. html). I took the role of Climbing Leader for the expedition. It was my fourth experience of Himalayan climbing. In short, the expedition to Shishapangma had a happy conclusion.

In October of the same year, 1990, AACK also sent another expedition to Meili Snow Mountain in Yunnan, China. Meili is a beautiful mountain located at the eastern end of the Great Himalaya Range (Fig. 1). Unfortunately, the Meili expedition ended in tragedy, with an avalanche accident on January 4, 1991. We lost 17 members, 11 Japanese and 6 Chinese climbers, in the disaster. At that time, I was in Africa. I visit Bossou, Guinea, West Africa, every year in December and January to study the wild chimpanzees living there (Matsuzawa et al. 2011). I was therefore not in a position to do anything at the time of the Meili avalanche accident.

In April 1994, 3 years after the accident, I travelled to Yunnan for the first time. I stayed in Kunming. The motivation for taking this trip was not climbing mountains but instead visiting places of interest and importance in 
Fig. 1 Meili Snow Mountain $(6740 \mathrm{~m})$ is located at the eastern end of the Great Himalaya Range. The Academic Alpine Club of Kyoto (AACK) made attempts to climb the untrodden peak in 1990 and then 1996. The highest peak is called Kawagebo (photo by Tetsuro Matsuzawa, February 22, 2014)

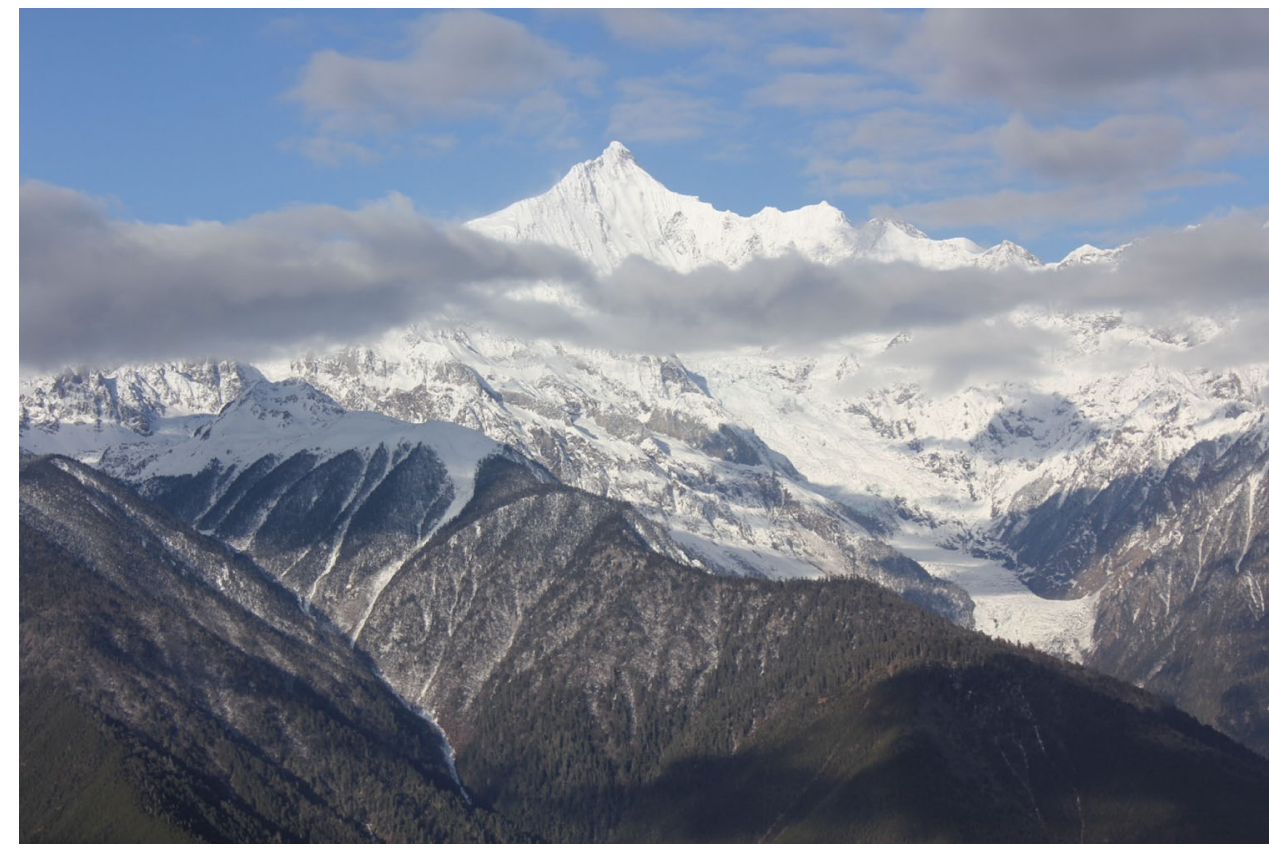

terms of cultural heritage; Yunnan is rich in cultural diversity as well as in biodiversity. After the 1991 Meili accident, I was seeking the opportunity to go to Yunnan. At that time, I had been focusing on cultural aspects of wild chimpanzees. Bossou chimpanzees use a pair of stones to crack open oil palm nuts. This unique behavior is a good example of a cultural tradition in wild chimpanzees. Consequently, I became interested in human culture as well. Thinking of human culture, I recognized that Yunnan is among the most interesting of places worldwide because of the variety of different ethnic groups there. There are 56 ethnic groups including the majority Han in China, with 26 represented in Yunnan. There are so-called minority ethnic groups, such as Yi, Bai, Dai, Lisu, Naxi, Hui, and Mongolian in Yunnan. I formed the idea of studying Mongolian traditions while in Yunnan. Mongolia is located to the north of China. Why, then, do Mongolians live in the southern part of China?

I organized a small expedition party comprised of four scholars majoring in primatology, pedagogy, Chinese philosophy, and ethics, respectively. We stayed in a Mongolian village, Xin Meng, near Kunming, for 8 days to form a rough sketch of the lifestyle of Mongolians living in Yunnan. In the Yuan dynasty, officially recorded to have been established by Chinggis Khan (Genghis Khan, 1162-1227), the country expanded in all directions including invasion to the south, Yunnan. When the empire began shrinking, the Mongolian soldiers and their families remained in Yunnan. My question was how did they maintain Mongolian cultural traditions for over 800 years? According to my observations and understanding, language and traditional dress play a key role in cultural identity.
Each ethnic group is distinguished by a unique language and costume. These are passed from one generation to the next.

I have continued exchanging information with scholars in Yunnan, such as Han Ning of Kunming University of Science and Technology. She acted as the English-Chinese translator when we visited Yunnan in 1994. At that time, she was an undergraduate student, but she got a job, subsequently, as faculty at Kunming University of Science and Technology. With the assistance of my Chinese friends, I have investigated the fauna of Yunnan because I passionately wanted to go back to Meili Snow Mountain. I focused on the region now called Three Parallel Rivers of Yunnan Protected Areas. This is a UNESCO World Natural Heritage Site. It lies within the drainage basins of the upper reaches of the Yangtze (Jinsha), Lancang (Mekong) and Nujiang (Salween) Rivers, the three largest rivers in Southeast Asia.

Close examination of the literature revealed an interesting picture of the distribution of snub-nosed monkeys. There are Yunnan snub-nosed monkeys living in the area between the Yangtze and the Mekong rivers. There is another species of Myanmar snub-nosed monkeys (Rhinopithecus strykeri, sp. nov.) living nearby the Salween River to the west (Geissmann et al. 2010). However, there are no records regarding the area between the Mekong and Salween Rivers. This area is a sort of missing link in our understanding of snub-nosed monkey distribution. Suppose that there was continuous distribution of similar species of the same genus Rhinopithecus: we would expect either an unknown species or past records of the missing link. The Meili Snow Mountain is located exactly 
within this missing link area. I came up with this idea and then talked with Long Yong-Cheng, the leading expert on Yunnan snub-nosed monkeys. We discussed the importance of a population survey of the potential "Meili snubnosed monkeys", and then organized a reconnaissance mission to assess existence of these unknown snub-nosed monkeys in the northwestern part of Yunnan.

The first expedition was carried out February to March 2014. The expedition members were the following five scholars: TM, Long Yong-Cheng, Han Ning, Liu Ning and his son, Liu Jie. We first went to observe Yunnan snubnosed monkeys. In Tachen, you can see a provisioned freeranging group of monkeys. It was my first encounter seeing the Yunnan snub-nosed monkeys in the wild. Their hair color was not gold colored, like golden snub-nosed monkeys in Sichuan, but black and white. We saw multi-male and multi-female parties there. They subsist on lichen (Fig. 2). I happened to witness a young monkey eat snow after eating lichen. The monkey repeated this behavior (Fig. 3a, b; supplementary video). I ate the lichen myself. I found it to be very dry. I suggest that the snow may help digestion of this dry food.

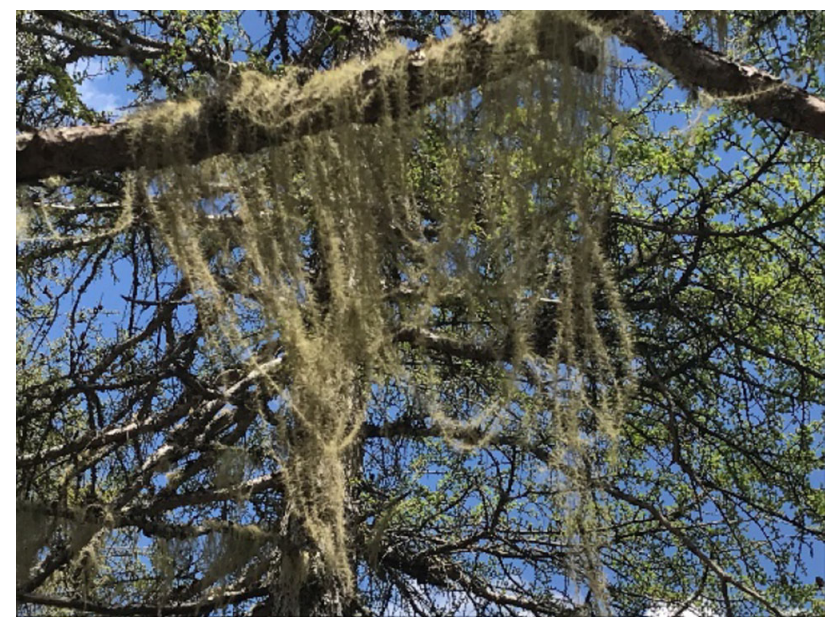

Fig. 2 Yunnan snub-nosed monkeys are characterized by their unique black and white hair color. The snub-nosed monkeys live on lichen (photo by Tetsuro Matsuzawa)
After watching the monkeys, we moved on to Meili Snow Mountain. We visited the memorial statue for the 1991 avalanche, built in Min Yong Village. Then, we travelled south down to the area between the Mekong and Salween Rivers, the area of the missing link of snub-nosed monkeys. We visited villages to get information on the snub-nosed monkeys and also went into the forests. We crossed the Salween River to the west bank. This area is very close to the border between China and Myanmar. We saw a single individual Myanmar snub-nosed monkey, rescued in the reserve center. The hair color of the Myanmar snub-nosed monkey was pure black.

The second expedition was carried out in April to May 2014. The three expedition members were the following: TM, Long Yong-Cheng, and Liu Jie. We first went to Laojun Mountain near the city Lijian (Fig. 4). We settled in at this location to carry out a long-term field study of Yunnan snub-nosed monkeys, as part of a larger project run by The Nature Conservancy (TNC). We wanted to know the behavior and ecology of the Yunnan snub-nosed monkeys in truly wild populations. Following the fieldwork, Liu Jie decided to join Kyoto University as a $\mathrm{PhD}$ student, starting in April 2016. Liu Jie continues to carry out field research on the Yunnan snub-nosed monkeys on Laojun Mountain.

The third expedition was carried out in September 2016. The five members were the following: TM, Satoshi Hirata, Ayane Inoue, Liu Jie and Liu Ning. We visited Meili Snow Mountain Base Camp. This was, once again, to look for the possible Meili snub-nosed monkeys. We used horses from the village to get to the Base Camp of Meili Snow Mountain (Fig. 5). We saw a lot of lichen that is the favorite food of snub-nosed monkeys on the way to the destination. However, we did not see any monkeys in the forests: no Meili snub-nosed monkeys on the mountain. We reached Meili Snow Mountain Base Camp, located at an altitude of about $4000 \mathrm{~m}$. It is a summer pasture for cattle. The climbing route of Meili Snow Mountain involves getting onto the glacier located at the east end. The melting glacier resulted in bare rock walls that necessitated difficult rock climbing.
Fig. 3 a, b I happened to witness a young monkey eat snow after eating lichen. The monkey repeated this behavior (see the supplementary video, taken by Tetsuro Matsuzawa)
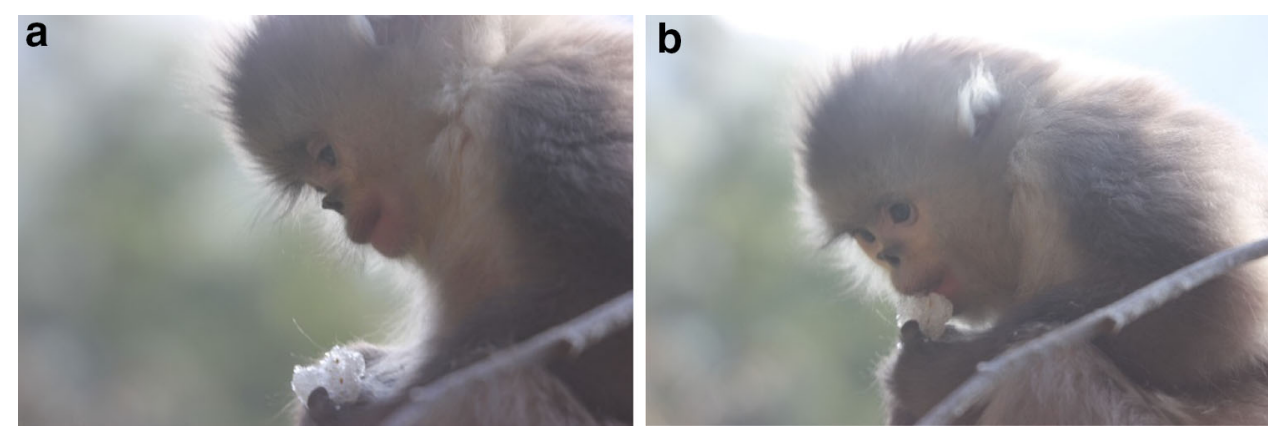


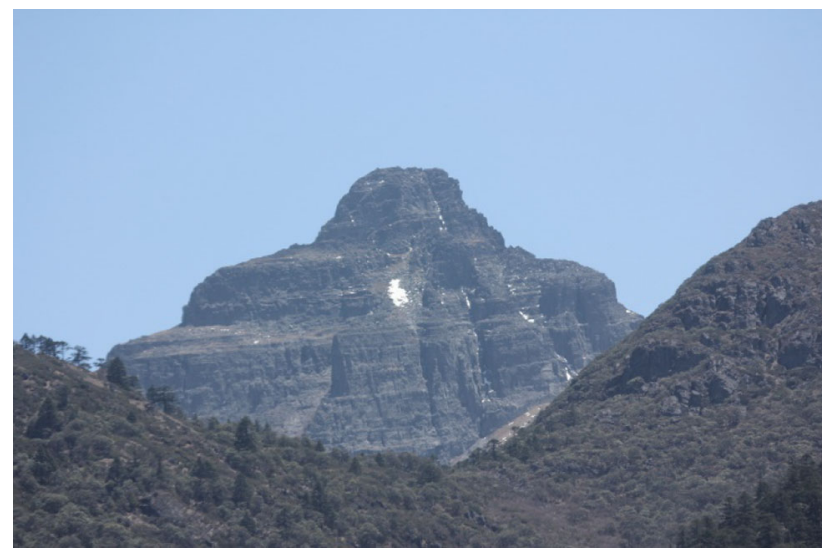

Fig. 4 Laojun Mountain near the city Lijian. This is the long-term field site for studying Yunnan snub-nosed monkeys (photo by Tetsuro Matsuzawa, May 2014)

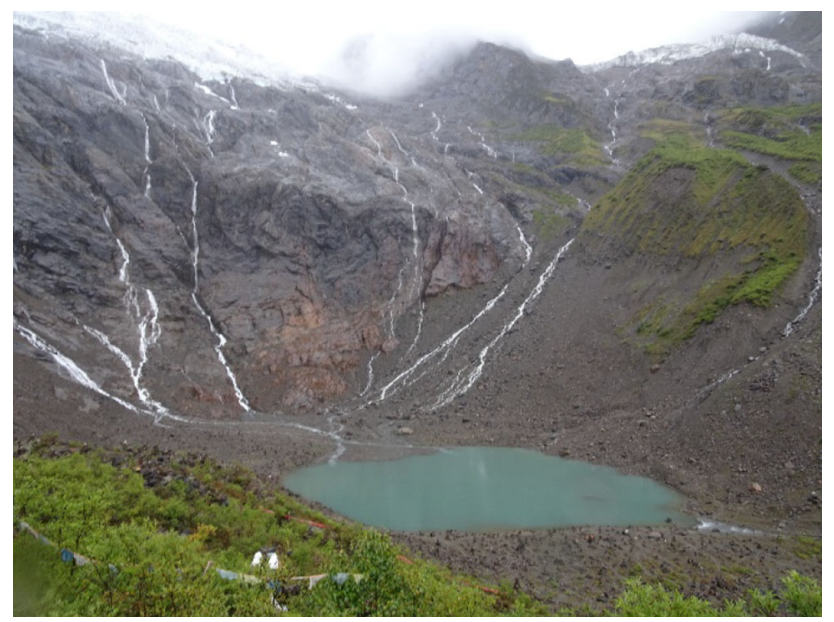

Fig. 5 We reached Meili Snow Mountain Base Camp, located at an altitude of about $4000 \mathrm{~m}$ and went higher to the end of the glacier. We looked for the possible Meili snub-nosed monkeys. We saw a lot of lichen that is the favorite food of snub-nosed monkeys on the way to the destination. However, we did not see any monkeys in the forests (photo by Tetsuro Matsuzawa)

The fourth expedition was carried out in April to May 2017. The members were the following four: TM, Ayane Inoue, Liu Jie, and Han Ning. We went to Laojun Mountain near Lijian again. This was to monitor the progress of Liu Jie's fieldwork on Yunnan snub-nosed monkeys at the long-term field site now established at Laojun Mountain. We spent the days looking for the monkeys and also climbed the 4000-m peak just above the ranging area of snub-nosed monkeys. Lichen is distributed within an altitude range of about $2800-3800 \mathrm{~m}$ in this area.

The main purpose of the visit was to use a drone for the first time in the field here. My colleagues and I have been using drones in fieldwork on various wild animals since 2016: wild chimpanzees in Bossou, Guinea; feral horses in Serra D'Arga, northern Portugal; wild dolphins in Ariake

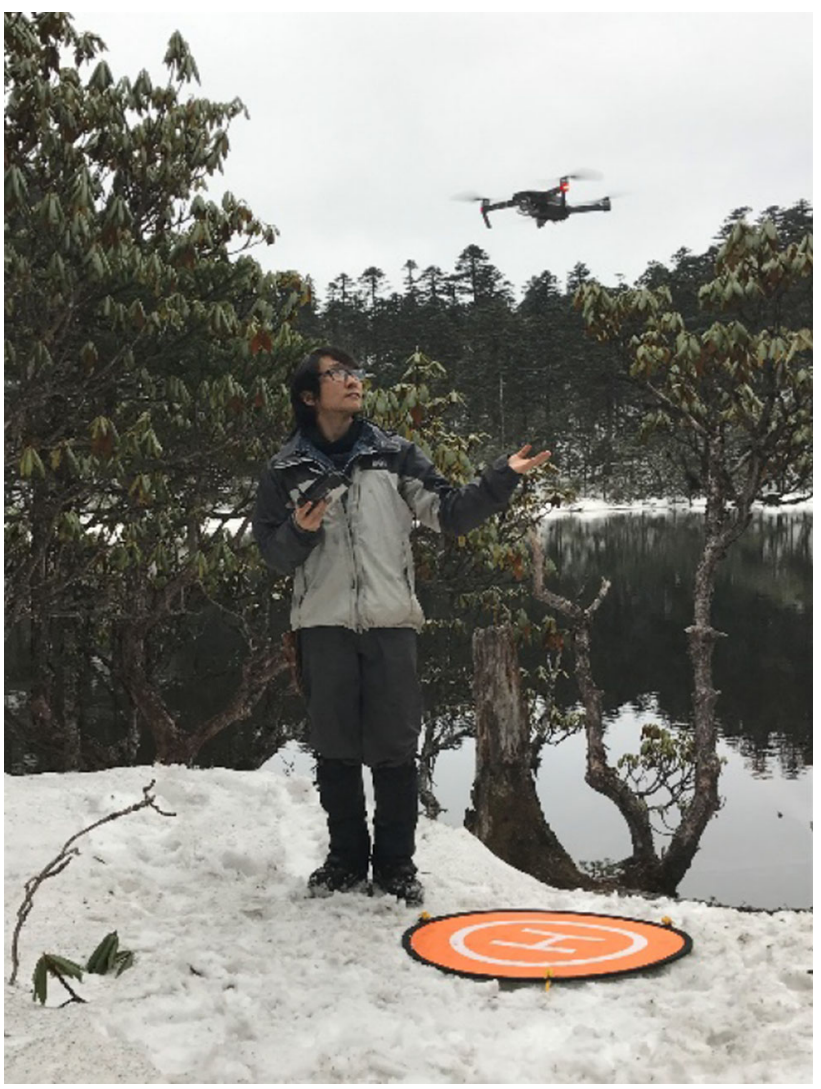

Fig. 6 Liu Jie launched many flights of the drone. It helped us to find the troop of snub-nosed monkeys (photo by Tetsuro Matsuzawa)

Bay, Japan. Liu Jie launched many flights of the drone (Fig. 6). In fact, it helped us to find the troop of snub-nosed monkeys. I believe we were the first to use a drone in the field to study wild snub-nosed monkeys.

Another purpose of the fourth expedition was to do a trial climb of the snow mountains in the Lijian area. There are two snow mountains near Lijian. One is Yulong Snow Mountain (5596 m), also called Jade Dragon Snow Mountain. Tourists can reach the high altitude of $4506 \mathrm{~m}$ by cable car to visit the Yulong Snow Mountain. At the time we visited, the mountain had attracted many tourists. Yulong Snow Mountain is renowned for having the glacier that is located at the most southerly end of the Great Himalaya Range: there are no more glaciers to the south. The mountain has various faces according to the direction from which it is viewed. We used a vehicle to move around the mountain and carried out reconnaissance to find the possible climbing route: from the northeast, the north, the west, and from the south: almost 270 degrees, rotating anticlockwise (Fig. 7). Only the view from the east was missing. Yulong is an untrodden peak. During this visit, I was made an honorary member of the Kunming Mountaineering Association. I hope that we will attempt to send a joint expedition to this untrodden peak in the near future. 


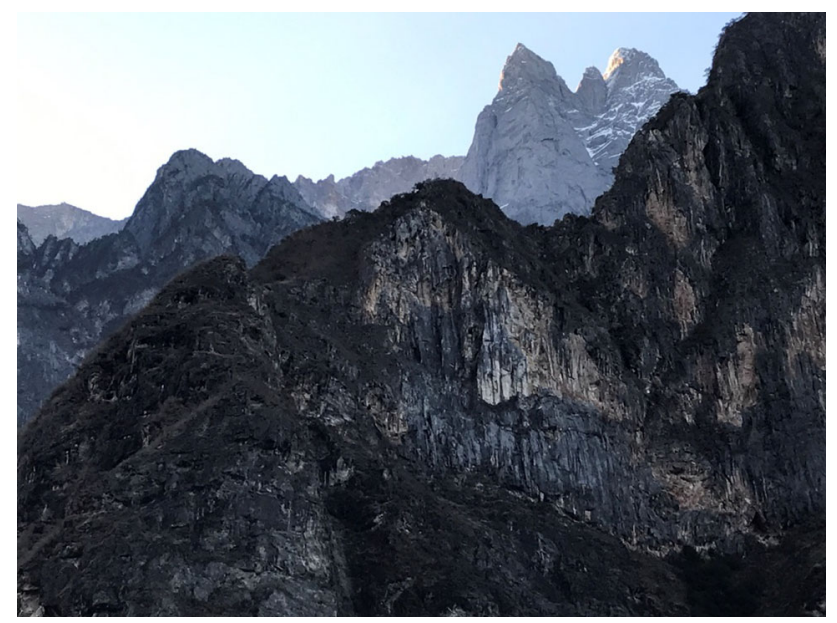

Fig. 7 The north face of Yulong Snow Mountain (5596 m), also called Jade Dragon Snow Mountain (photo by Tetsuro Matsuzawa)

Another mountain in Lijian is Haba Snow Mountain (5396 m). The mountain looks much easier to climb than any other snow peak in Yunnan. We rode on mules from Haba Village at an altitude of $2535 \mathrm{~m}$. We reached a place just below Haba Snow Mountain Base Camp: the altitude here was $3635 \mathrm{~m}$ (Fig. 8). The mules took us up and down more than $1000 \mathrm{~m}$ of altitude in 1 day. Haba Snow Mountain may provide a good opportunity for climbers seeking to acclimatize to high altitudes and to do basic skills training in preparation for climbing new mountains. There was lichen in the forest of Haba, but very little. There was no sign of the snub-nosed monkeys. The climate

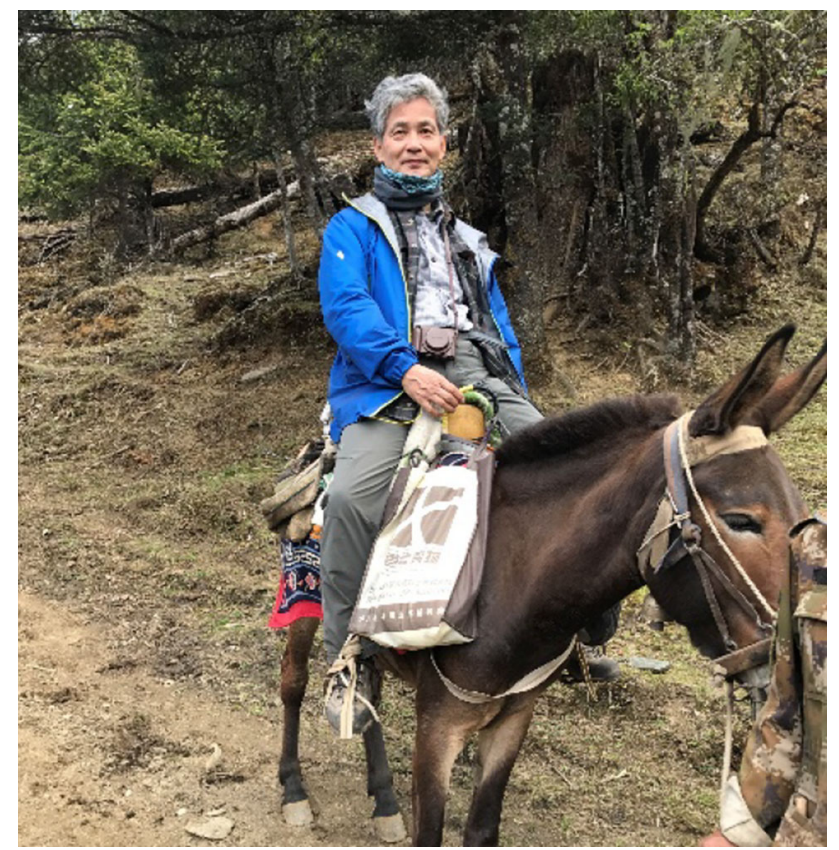

Fig. 8 We rode on muleback from Haba Village to an altitude of 3635 m on Haba Snow Mountain (5396 m) (photo by Liu Jie) appeared too dry for the monkeys, or to support forests with lichen.

There is a big river, Yangtze River, between the two snow mountains, Yulong and Haba. The narrowest part is a rapid stream called Tiger Leaping Gorge. The configuration of the two enormous mountains with the river in between reminded me of another such case in the Great Himalaya Range. Brahmaputra River separates Namcha Barwa $(7782 \mathrm{~m})$ and Gyala Peri $(7294 \mathrm{~m})$. Both cases provide us with the dynamic and beautiful scene of snowy mountains and rivers. The old town of Lijiang is designated as a UNESCO World Cultural Heritage Site. The Three Parallel Rivers of Yunnan Protected Area, including Laojun, Yulong, and Haba mountains, is designated as a UNESCO World Natural Heritage Site. I believe that future pioneering efforts on monkeys and mountains in this area will show us the beauty of Yunnan, exceptional in terms of its mixture of both nature and culture.

Acknowledgement This article is based on four field expeditions to Yunnan from 2014 to 2017. The study was financially supported by MEXT 16H06283, JSPS core-to-core CCSN, and JSPS leading graduate program U04 (PWS) to TM. Thanks are due to The Nature Conservancy (TNC), the local authority of Laojun Mountain National Park, and also to my colleagues: Long Yong-Cheng, Han Ning, Liu Ning, Liu Jie, and Ayane Inoue.

\section{References}

Geissmann T, Lwin N, Aung Saw Soe, Aung Thet Naing, Aung Zin Myo, Hla Tony Htin, Grindley Mark, Momberg Frank (2010) A new species of snub-nosed monkey, genus Rhinopithecus MilneEdwards, 1872 (Primates, Colobinae), from northern Kachin state, northeastern Myanmar. Am J Primatol 73(1):96-107. doi:10.1002/ajp.20894

Long YC, Kirkpatrick CR (1994) Report on the distribution, population, and ecology of the Yunnan snub-nosed monkey (Rhinopithecus bieti). Primates 35(2):241-250

Matsuzawa T (2016) Mountain day: isomorphism of mountaineering and science. Primates 57(4):441-444

Matsuzawa T, Humle T, Sugiyama Y (2011) The chimpanzees of Bossou and Nimba. Springer, New York

Qi XG, Garber PA, Ji W, Huang ZP, Huang K, Zhang P, Guo ST, Wang XW, He G, Zhang P, Li BG (2014) Satellite telemetry and social modeling offer new insights into the origin of primate multilevel societies. Nat Commun. doi:10.1038/ncomms6296

Zhang P, Watanabe K, Li B, Tan CL (2006) Social organization of Sichuan snub-nosed monkeys (Rhinopithecus roxellana) in the Qinling Mountains, Central China. Primates 47(4):374-382 